\begin{tabular}{|c|c|}
\hline & DJS Vol. 41 (2020) 20-29 \\
\hline 1969 & $\begin{array}{c}\text { Delta Journal of Science } \\
\text { Available online at } \\
\text { https://djs.journals.ekb.eg/ }\end{array}$ \\
\hline Research Article & \\
\hline
\end{tabular}

\title{
Right Truncated Fréchet-Weibull Distribution: Statistical Properties and Application
}

\author{
Abd El-monem A. M. Teamah, Ahmed A. Elbanna and Ahmed M. Gemeay \\ Department of Mathematics, Faculty of Science, Tanta University, Tanta, Egypt
}

\begin{abstract}
A right truncated distribution called Right Truncated Fréchet -Weibull distribution with its mathematical forms of density, cumulative, survival and reverse hazard functions were provided in this paper. Statistical properties such as moments, quantile function, mode and moment generating function were studied as well as obtaining special cases to other truncated distributions. Maximum likelihood method used to estimate parameters numerically for randomly generated dataset and real-world data.
\end{abstract}

Key words: Fréchet distribution, Weibull distribution, Survival function, Moments, Quantile function, Moment Generating function, Order statistics, Entropy.

\section{Introduction:}

Results of studies that are based on the entire population cannot be applied on only a particular class of the society that possesses specific characteristics. The results of such studies will be incorrect as a result of the negative impact of specific elements of society on the study group. In any case, putting such conditions on the society, the data would not follow a similar distribution of whole society.

Then, we have to determine the distributional attributes of the truncated data including the probability density functions. Truncated distributions are conditional distributions obtained when we reduce the domain of original distribution to a smaller one. Truncation is used when there is no ability to know the events occurring above or below the studied phenomenon such as the study of plant growth, which cannot be studied before the growth of the plant over the soil, so that the truncated distributions have an important role in various fields such as agriculture, medicine, engineering, physics,...etc. But in that article we use a method to overcome the extreme values in the data by putting the right truncated value of our distribution equal upper whisker limit.

M.M. Ali and N. Saralees [2] considered that the popular long-tailed distribution is Pareto distribution and they presented a truncated version to overcome its long tailed. 
L. Zaninetti [10] presented a right and left truncated gamma distribution with application to the stars that introduces an upper and a lower boundary to this distribution. The parameters which characterize the truncated gamma distribution were evaluated. A statistical test is performed on two samples of stars. A comparison with the log-normal and the four power law distribution is made. J.T. Hattaway [7] studied the parameter estimation and hypothesis testing for the truncated normal distribution with applications to introductory statistics grades. S.H. Abid [1] considered a doubly-truncated Fréchet random variable restricted by both a lower (c) and upper (d) truncation points provided with some statistical properties and different methods to estimate distribution parameters were evaluated. M. El-Din et al. [3] derived the probability density function of mid truncated distribution and used Lindley distribution as illustrative example. Finally, the pdf of sum of mid truncated Lindley distribution is obtained. Z. Behdani [2] introduced some properties and characterization of inequality measures and truncated distributions along with relationships between truncated and original variables in the context of reliability and economics measures.

The probability density function (PDF) and the cumulative distribution function (CDF) for the Fréchet-Weibull random variable $X>$ 0 are given by

$$
\begin{gathered}
f(x)=\alpha k \beta^{\alpha} \lambda^{\alpha k} x^{-1-\alpha k} \exp \left(-\beta^{\alpha}\left(\frac{\lambda}{x}\right)^{\alpha k}\right), \\
F(x)=\exp \left(-\beta^{\alpha}\left(\frac{\lambda}{x}\right)^{\alpha k}\right),
\end{gathered}
$$

respectively, where $\alpha$ and $k$ are shape parameters, and $\lambda$ and $\beta$ are scale parameters.

This paper is organized as follows. In section 2 we introduce Right Truncated FréchetWeibull Distribution represented by its PDF and CDF along with survival function, hazard function, reverse hazard function, The effect of the parameters on PDF, CDF, $\mathrm{S}(\mathrm{x})$ and $\mathrm{h}(\mathrm{x})$, and special cases of our distribution. Statistical properties such as moments, coefficients of skewness, kurtosis and variation, quantile function, mode, moment generating function, mean deviation about mean, several entropy types such as Renyi, Tsallis and Shannon entropies, and mathematical Lorenz and Bonferroni curves are in section 3 . In section 4 we introduce order statistics, its probability and cumulative function, and limit distribution of its maximum. Estimation of Right Truncated Fréchet-Weibull distribution's parameters by maximum likelihood method is in Section 5 . In section 6 we estimate parameters of randomly generated data and then we extend the application to real-world data.

\section{Right Truncated Fréchet -Weibull Distribution (RTFWD)}

A random variable $\mathrm{X}$ is said to have RTFWD with four parameters $\alpha, \beta, \lambda, k$, where its cumulative distribution function (CDF) for $0<x \leq b$ is defined as

$$
F(x)=\frac{G(x)}{G(b)}=\exp \left(-\beta^{\alpha} \lambda^{\alpha k}\left(x^{-\alpha k}-b^{-\alpha k}\right)\right),
$$

and its probability density function $(\mathrm{PDF})$ is given by

$$
\begin{aligned}
f(x) & =\alpha k \beta^{\alpha} \lambda^{\alpha k} x^{-1-\alpha k} \\
& \times \exp \left(-\beta^{\alpha} \lambda^{\alpha k}\left(x^{-\alpha k}-b^{-\alpha k}\right)\right)
\end{aligned}
$$

where $\alpha$ and $k$ are shape parameters, $\lambda$ and $\beta$ are scale parameters, and $\mathrm{G}(\mathrm{x})$ is CDF of Fréchet-Weibull distribution.

\subsection{Survival function}

The survival function (reliability function) of RTFWD is given by

$S(x)=1-F(x)=1-\exp \left(-\beta^{\alpha} \lambda^{\alpha k}\left(x^{-\alpha k}-b^{-\alpha k}\right)\right)$.

\subsection{Hazard function}

The hazard function of RTFWD is given by

$$
\begin{aligned}
h(x) & =\frac{f(x)}{S(x)}=\alpha k \beta^{\alpha} \lambda^{\alpha k} x^{-1-\alpha k} \\
& \times \frac{\exp \left(-\beta^{\alpha} \lambda^{\alpha k}\left(x^{-\alpha k}-b^{-\alpha k}\right)\right)}{1-\exp \left(-\beta^{\alpha} \lambda^{\alpha k}\left(x^{-\alpha k}-b^{-\alpha k}\right)\right)} .
\end{aligned}
$$




\subsection{Reverse hazard function}

The reverse hazard function of RTFWD is given by

$$
r(x)=\frac{f(x)}{F(x)}=\alpha k \beta^{\alpha} \lambda^{\alpha k} x^{-1-\alpha k} .
$$

\subsection{The effect of the parameters on $\mathrm{CDF}, \mathrm{PDF}, \mathrm{S}(\mathrm{x})$ and $\mathrm{h}(\mathrm{x})$ of RTFWD}

Plots of PDF (2), CDF (1), S(x) (3) and h(x) (4) of RTFWD are displayed in figures 1, 2, 3 and 4 for different parameter values, respectively.

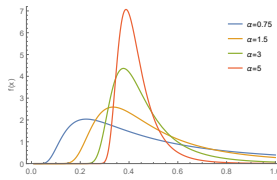

(a)

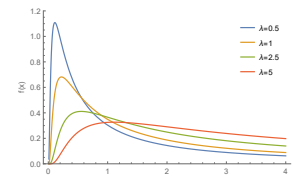

(b)
Figure 1: PDF plots for RTFWD

Figure (1a) shows how PDF behave, affected by the change of parameter $\alpha$, where $\mathrm{b}=1, \beta=0.7, \lambda=0.5$ and $k=1.5$, while figure (1b) shows the behavior of PDF by changing the parameter $\lambda$, where $\mathrm{b}=4, \alpha=0.5$, $\beta=2$ and $k=1$.

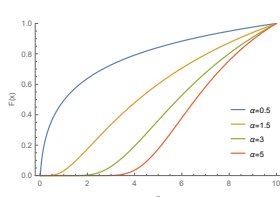

(a)

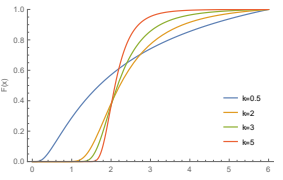

(b)
Figure 2: CDF plots for RTFWD

Figure (2a) shows how CDF behave, affected by the change of parameter $\alpha$, where $\mathrm{b}=10, \beta=0.5, \lambda=3$ and $k=0.5$, while figure (2b) shows the behavior of CDF by changing the parameter parameter $\mathrm{k}$, where $\mathrm{b}=6$, $\alpha=1.5, \beta=1$ and $\lambda=2$.

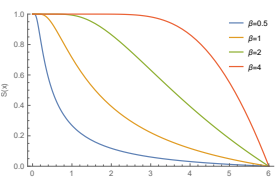

(a)

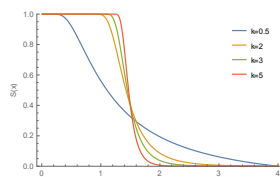

(b)
Figure 3: S(x) plots for RTFWD

Figure (3a) shows how $\mathrm{S}(\mathrm{x})$ behave, affected by the change of parameter parameter $\beta$, where $\mathrm{b}=6, \alpha=2, \lambda=1.5$ and $k=0.5$, while figure (3b) shows the behavior of $\mathrm{S}(\mathrm{x})$ by changing the parameter $k$, where $\mathrm{b}=4, \alpha=3$, $\beta=0.8$ and $\lambda=1.5$.

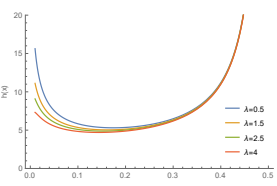

(a)

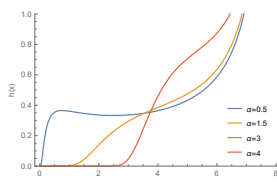

(b)
Figure 4: Plots of the RTFWD $\mathrm{h}(\mathrm{x})$ for some parameter values

Figure (4a) shows how $\mathrm{h}(\mathrm{x})$ behave, affected by the change of parameter $\lambda$, where $\mathrm{b}=0.5$, $\alpha=0.5, \beta=1$ and $k=0.5$, while figure (4b) shows the behavior of $\mathrm{h}(\mathrm{x})$ by changing the parameter $\alpha$, where $\mathrm{b}=4, \beta=3, \lambda=1.5$ and $k=1$. The behavior of $\mathrm{h}(\mathrm{x})$ can be unimodal, decreasing or increasing depending on the values of parameters.

\subsection{Special cases for RTFWD}

If $\mathrm{X}$ is a random variable with $\mathrm{CDF}$ in equation (1), then we have the following special cases:

- When $\lambda=1$ and $k=1$, then equation (1) reduces to give Right Truncated Fréchet Distribution with the following CDF :

$F(x)=\exp \left(-\beta^{\alpha}\left(x^{-\alpha}-b^{-\alpha}\right)\right), 0<x \leq b$.

- When $\beta=1, \alpha=1$ and $k=1$, then equation (1) reduces to give Right Truncated Inverse Exponential Distribution with the following CDF:

$$
F(x)=\exp \left(-\lambda\left(x^{-1}-b^{-1}\right)\right), 0<x \leq b .
$$


- When $\alpha=1, \lambda=1$ and $k=2$, then equation (1) reduces to give Right Truncated Inverse Raylieh Distribution with the following CDF:

$$
F(x)=\exp \left(-\beta\left(x^{-2}-b^{-2}\right)\right), 0<x \leq b .
$$

- When $\lambda=1$ and $\alpha=1$, then equation (1) reduces to give Right Truncated Inverse Weibull Distribution with the following CDF:

$$
F(x)=\exp \left(-\beta\left(x^{-k}-b^{-k}\right)\right), 0<x \leq b .
$$

- When $\alpha=1$, then equation (1) reduces to give Right Truncated Generalized Inverse Weibull Distribution with the following CDF:

$F(x)=\exp \left(-\beta \lambda^{k}\left(x^{-k}-b^{-k}\right)\right), 0<x \leq b$.

\section{Statistical Properties}

\subsection{Moments}

The $r^{t h}$ moments $\mu_{r}^{\prime}$ about the origin of RTFWD is given by

$$
\mu_{r}^{\prime}=E\left(x^{r}\right)=\lambda^{r} \beta^{\frac{r}{k}} p g(r), r<\alpha k,
$$

where $g(r)=\Gamma\left(1-\frac{r}{\alpha k}, \beta^{\alpha}\left(\frac{\lambda}{b}\right)^{\alpha k}\right)$ is upper incomplete gamma function and $p=$ $\exp \left(\beta^{\alpha} \lambda^{\alpha k} b^{-\alpha k}\right)$. By setting $\mathrm{r}=1,2,3$ and 4 we obtain the first four moments about the origin of RTFWD, respectively.

Mean and variance of RTFWD are as given by:

$$
\begin{aligned}
\mu_{1}^{\prime} & =\mu=\lambda \beta^{\frac{1}{k}} p g(1), \\
\sigma^{2} & =\beta^{2 / k} \lambda^{2} p\left(g(2)-g(1)^{2} p\right),
\end{aligned}
$$

respectively. The central moments about the mean of RTFWD can be obtained by the following relations

$$
\begin{aligned}
\mu_{1} & =\mu_{1}^{\prime}-\mu=0, \\
\mu_{2} & =\mu_{2}^{\prime}-\left(\mu_{1}^{\prime}\right)^{2}=\beta^{2 / k} \lambda^{2} p\left(g(2)-g(1)^{2} p\right), \\
\mu_{3} & =\mu_{3}^{\prime}-3 \mu_{2}^{\prime} \mu_{1}^{\prime}+2\left(\mu_{1}^{\prime}\right)^{3} \\
& =\beta^{3 / k} \lambda^{3} p\left(2 g(1)^{3} p^{2}-3 g(2) g(1) p+g(3)\right), \\
\mu_{4} & =\mu_{4}^{\prime}-4 \mu_{3}^{\prime} \mu_{1}^{\prime}+6 \mu_{2}^{\prime}\left(\mu_{1}^{\prime}\right)^{2}-3\left(\mu_{1}^{\prime}\right)^{4} \\
& =-\beta^{4 / k} \lambda^{4} p\left(3 g(1)^{4} p^{3}-6 g(2) g(1)^{2} p^{2}\right. \\
& +4 p g(3) g(1)-g(4)),
\end{aligned}
$$

respectively, which will be used in section (3.2).

\subsection{Coefficients of Skewness, Kurtosis and Variation}

\subsubsection{Coefficient of Skewness}

The coefficient of skewness is a method to determine the skewness of the distribution and it can be obtained for RTFWD by the following relation

$\beta_{1}=\frac{\left(\mu_{3}\right)^{2}}{\left(\mu_{2}\right)^{3}}=-\frac{\left(2 g(1)^{3} p^{2}-3 g(2) g(1) p+g(3)\right)^{2}}{p\left(g(1)^{2} p-g(2)\right)^{3}}$.

\subsubsection{Coefficient of Kurtosis}

Coefficient of kurtosis is a way to determine the distribution curve whether is leptokurtic curve or platykurtic curve relative to a normal distribution and it can be obtained for RTFWD by the following relation

$$
\begin{aligned}
\beta_{2} & =\frac{\mu_{4}}{\left(\mu_{2}\right)^{2}} \\
& =\frac{g(1) p\left(-3 g(1)^{3} p^{2}+6 g(2) g(1) p-4 g(3)\right)+g(4)}{p\left(g(2)-g(1)^{2} p\right)^{2}} .
\end{aligned}
$$

\subsubsection{Coefficient of Variation}

The coefficient of variation $(\mathrm{CV})$ is a method to determine the dispersion of the distribution, it is also used to compare between distributions and it can be obtained for RTFWD by the following relation

$$
C V=\frac{\sigma}{\mu} \times 100=\frac{\sqrt{p\left(g(2)-g(1)^{2} p\right)}}{g(1) p} \times 100 .
$$

\subsection{Quantile function}

The quantile function (inverse CDF) $Q(p)$ of RTFWD is given by

$$
\begin{aligned}
Q(p) & =\inf \{x \in R: F(x) \geq p\} \\
& =\left(b^{-\alpha k}-\beta^{-\alpha} \lambda^{-\alpha k} \ln p\right)^{\frac{-1}{\alpha k}}
\end{aligned}
$$

where $0<p \leq 1$. By setting $\mathrm{p}=0.25,0.5$ and 0.75 we obtained the first, second and third quartiles of RTFWD, respectively. 


\subsection{Mode}

Mode is the most happening value between data. Consider the PDF of the RTFWD given in (2), by taking the logarithm of this PDF, by differentiating it with respect to $\mathrm{x}$ and setting it equal to zero, we get the mode as following

$$
x_{0}=\lambda\left(\frac{\alpha k \beta^{\alpha}}{1+\alpha k}\right)^{\frac{1}{\alpha k}} .
$$

\subsection{Moment generating function}

The moment generating function of RTFWD is given by

$$
\begin{aligned}
M(t) & =\int_{x=0}^{b} \exp (t x) f(x) d x=\exp \left(\beta^{\alpha}\left(\frac{\lambda}{b}\right)^{\alpha k}\right) \\
& \times \sum_{m=0}^{\infty} \frac{t^{m}}{m !} \lambda^{m} \beta^{\frac{m}{k}} \Gamma\left(1-\frac{m}{\alpha k}, \beta^{\alpha}\left(\frac{\lambda}{b}\right)^{\alpha k}\right),
\end{aligned}
$$

where $\Gamma\left(1-\frac{m}{\alpha k}, \beta^{\alpha}\left(\frac{\lambda}{b}\right)^{\alpha k}\right)$ is upper incomplete gamma function.

The characteristics function $\phi(t)$ for RTFWD is obtained by

$$
\begin{aligned}
\phi(t) & =\exp \left(\beta^{\alpha}\left(\frac{\lambda}{b}\right)^{\alpha k}\right) \\
& \times \sum_{m=0}^{\infty} \frac{(i t)^{m}}{m !} \lambda^{m} \beta^{\frac{m}{k}} \Gamma\left(1-\frac{m}{\alpha k}, \beta^{\alpha}\left(\frac{\lambda}{b}\right)^{\alpha k}\right) .
\end{aligned}
$$

\subsection{Mean residual life function}

The mean residual life function of a continuous random variable $\mathrm{T}$ and survival function $S(t)$ is given by

$$
\mu(t)=E(T-t \mid T>t)=\frac{1}{S(t)} \int_{t}^{\infty} S(u) d u .
$$

The mean residual life function of RTFWD is obtained by

$$
\begin{aligned}
\mu(x) & =b \frac{S(b)}{S(x)}-x+\frac{\beta^{\frac{1}{k}} \lambda \exp \left(\beta^{\alpha}\left(\frac{\lambda}{b}\right)^{\alpha k}\right)}{S(x)} \\
& \times \int_{\beta^{\alpha}\left(\frac{\lambda}{b}\right)^{\alpha k}}^{\beta^{\alpha}\left(\frac{\lambda}{x}\right)^{\alpha k}} w^{\frac{-1}{\alpha k}} e^{-w} d w .
\end{aligned}
$$

\subsection{Mean deviation}

The mean deviation (MD) is the absolute expected deviations of data from any measures of central tendency.

\subsubsection{Mean deviation about the mean}

The mean deviation about the mean of RTFWD is given by

$$
\begin{aligned}
M D & =\int_{x=0}^{b}|x-\mu| f(x) d x \\
& =2 \mu(F(\mu)-1)+2 \beta^{\frac{1}{k}} \lambda \exp \left(\beta^{\alpha}\left(\frac{\lambda}{b}\right)^{\alpha k}\right) \\
& \times \int_{\exp \left(\beta^{\alpha}\left(\frac{\lambda}{b}\right)^{\alpha k}\right)}^{\exp \left(\beta^{\alpha}\left(\frac{\lambda}{\mu}\right)^{\alpha k}\right)} y^{\frac{-1}{\alpha k}} e^{-y} d y .
\end{aligned}
$$

Similarly, the mean deviation about the median or any result of another measure of central tendency can be obtained by replacing $\mu$ in previous equation by another measure of central tendency.

\subsection{Entropy}

Information theory has mathematical origin in entropy notion that is related to thermodynamic and statistical mechanics. In 1948, the definition of shannon entropy was introduced. After 1948, variate extensions of the Shannon entropy has been introduced such as Renyi entropy (1961), and Tsallis entropy (1988).(for more information see [9])

\subsubsection{Renyi entropy}

The continuous renyi entropy of RTFWD is defined as

$$
\begin{aligned}
R_{r}(x) & =\frac{1}{1-r} \log \int_{x=0}^{b} f^{r}(x) d x, r>0, r \neq 1 \\
& =\frac{1}{1-r} \log \left[(\alpha k)^{r-1} \lambda^{1-r} \beta^{\frac{1}{k}(1-r)}\right. \\
& \times r^{\frac{1}{\alpha k}-\frac{r}{\alpha k}-r} \exp \left(r \beta^{\alpha}\left(\frac{\lambda}{b}\right)^{\alpha k}\right) \\
& \left.\times \Gamma\left(\frac{r}{\alpha k}+r-\frac{1}{\alpha k}, r \exp \left(\beta^{\alpha}\left(\frac{\lambda}{b}\right)^{\alpha k}\right)\right)\right]
\end{aligned}
$$

where $\Gamma\left(\frac{r}{\alpha k}+r-\frac{1}{\alpha k}, r \exp \left(\beta^{\alpha}\left(\frac{\lambda}{b}\right)^{\alpha k}\right)\right)$ is upper incomplete gamma function.

\subsubsection{Tsallis entropy}

The continuous tsallis entropy of RTFWD is defined as

$$
T_{r}(x)=-R_{r}(X)-1 \quad, r>0, r \neq 1 .
$$




\subsubsection{Shannon entropy}

The continuous shannon entropy of RTFWD is defined as

$$
S_{H}(x)=-\int_{x=0}^{b} f(x) \log f(x) d x,
$$

where Shannon entropy is aspecial case of Renyi entropy when $\mathrm{r}$ tends to 1 .

$$
\begin{aligned}
S_{H}(x) & =\lim _{r \rightarrow 1} R_{r}(x)=1+\log \left(\frac{\lambda \beta^{\frac{1}{k}}}{\alpha k}\right)-\beta^{\alpha}\left(\frac{\lambda}{b}\right)^{\alpha k} \\
& \times\left(1-\exp \left(\beta^{\alpha}\left(\frac{\lambda}{b}\right)^{\alpha k}\right)\right)-\left(1+\frac{1}{\alpha k}\right) \\
& \times \exp \left(\beta^{\alpha}\left(\frac{\lambda}{b}\right)^{\alpha k}\right) \int_{\beta^{\alpha}\left(\frac{\lambda}{b}\right)^{\alpha k}}^{\infty} e^{-u} \ln (u) d u .
\end{aligned}
$$

\subsection{Lorenz and Bonferroni curves}

The Lorenz curve $L_{p}(x)$ was defined for a continuous random variable $\mathrm{X}(X>0)$ by the following relation

$$
L_{x}(p)=\frac{1}{\mu} \int_{x=0}^{x_{p}} x f(x) d x,
$$

where $f(x)$ is the corresponding PDF of $X, \mu$ is the mean of $\mathrm{X}$ and $x_{p}$ is the quantile function such that $F\left(x_{p}\right)=p$ (for more details see $[5]) . L(0)=0, L(1)=1$ and Lorenz curve is undefined if mean equal zero.

The Lorenz curve of RTFWD is given by

$$
\begin{aligned}
L_{x}(p) & =\frac{1}{\Gamma\left(1-\frac{1}{\alpha k}, \beta^{\alpha}\left(\frac{\lambda}{b}\right)^{\alpha k}\right)} \\
& \times \int_{\beta^{\alpha} \lambda^{\alpha k}\left(b^{-\alpha k}-\beta^{-\alpha} \lambda^{-\alpha k} \ln p\right)}^{\infty} y^{\frac{-1}{\alpha k}} e^{-y} d y .
\end{aligned}
$$

Bonferroni curve is defined as (for more details see [8])

$$
B_{x}(p)=\frac{1}{\mu F(x)} \int_{0}^{x_{p}} x f(x) d x=\frac{L_{x}(p)}{F(x)} .
$$

\section{Order statistics}

\subsection{Probability and cumulative function}

Let $X_{1}, X_{2}, \ldots, X_{n}$ is a random sample from TFW distribution. Let $X_{1: n}<X_{2: n}<\ldots<$
$X_{n: n}$ denote the corresponding order statistics. The probability density and the cumulative distribution functions of the ith-order statistic of RTFWD are given by

$$
\begin{aligned}
f_{i: n}(x) & =\frac{n !(F(x))^{i-1}(1-F(x))^{n-i} f(x)}{(i-1) !(n-i) !} \\
& =\frac{n ! \alpha k \beta^{\alpha} \lambda^{\alpha k} x^{-1-\alpha k}}{(i-1) !(n-i) !} \\
& \times\left(\exp \left(-\beta^{\alpha} \lambda^{\alpha k}\left(x^{-\alpha k}-b^{-\alpha k}\right)\right)\right)^{i} \\
& \times\left(1-\exp \left(-\beta^{\alpha} \lambda^{\alpha k}\left(x^{-\alpha k}-b^{-\alpha k}\right)\right)\right)^{n-i}, \\
F_{i: n}(x) & =\sum_{r=i}^{n}\left(\begin{array}{l}
n \\
r
\end{array}\right)(F(x))^{r}(1-F(x))^{n-r} \\
& =\sum_{r=i}^{n}\left(\begin{array}{l}
n \\
r
\end{array}\right)\left(\exp \left(-\beta^{\alpha} \lambda^{\alpha k}\left(x^{-\alpha k}-b^{-\alpha k}\right)\right)\right)^{r} \\
& \times\left(1-\exp \left(-\beta^{\alpha} \lambda^{\alpha k}\left(x^{-\alpha k}-b^{-\alpha k}\right)\right)\right)^{n-r},
\end{aligned}
$$

respectively, by setting $i=1$ we obtain the distribution of minimum order statistics and by setting $i=n$ we obtain the distribution of maximum order statistics of RTFWD.

\subsection{Limiting distribution for maximum order statistics}

Suppose that $Z_{n}=X_{n: n}=$ $\max \left(X_{1}, X_{2}, \ldots, X_{n}\right)$ from RTFWD and the limiting distribution of $Z_{n}$ can be obtained by the theorem (2.1.2) in [6]

$$
\lim _{x \rightarrow+\infty} \frac{1-F\left(b-\frac{1}{t x}\right)}{1-F\left(b-\frac{1}{t}\right)}=x^{-1},
$$

$\lim _{n \rightarrow+\infty} P\left(Z_{n}<a_{n}+b_{n} x\right)=\left\{\begin{array}{ll}1 & \text { if } x \geqslant 0 \\ \exp (x) & \text { if } x<0\end{array}\right.$, and the normalizing constants are $a_{n}=b$ and $b_{n}=b-\left(b^{-\alpha k}-\beta^{-\alpha} \lambda^{-\alpha k} \ln \left(1-\frac{1}{n}\right)\right)^{\frac{-1}{\alpha k}}$.

\section{Parameters estimation}

For estimating the parameters of RTFWD we use maximum likelihood estimation. Let $X=$ $\left(x_{1}, x_{2}, \ldots, x_{n}\right)$ be independent random sample having probability density function (2), then the likelihood function is given by 


$$
\begin{aligned}
L(x) & =\alpha^{n} k^{n} \beta^{n \alpha} \lambda^{n \alpha k} \exp \left(n \beta^{\alpha}\left(\frac{\lambda}{b}\right)^{\alpha k}\right) \\
& \times \exp \left(-\beta^{\alpha} \lambda^{\alpha k} \sum_{i=1}^{n} x_{i}^{-\alpha k}\right) \prod_{i=1}^{n} x_{i}^{-1-\alpha k},
\end{aligned}
$$

by taking logarithm, we find the log-likelihood function as

$$
\begin{aligned}
\log L(x) & =n[\log \alpha+\log k+\alpha \log \beta+\alpha k \log \lambda \\
& \left.+\left(\frac{\beta \lambda^{k}}{b^{k}}\right)^{\alpha}\right]-\beta^{\alpha} \lambda^{\alpha k} \sum_{i=1}^{n} x_{i}^{-\alpha k} \\
& -(1+\alpha k) \sum_{i=1}^{n} \log x_{i}
\end{aligned}
$$

From equation (7), we get

$$
\begin{aligned}
\frac{\partial \log L(x)}{\partial \alpha} & =n\left[\frac{1}{\alpha}+\log \beta \lambda^{k}+\left(\frac{\beta \lambda^{k}}{b^{k}}\right)^{\alpha} \log \frac{\beta \lambda^{k}}{b^{k}}\right] \\
& -k \sum_{i=1}^{n} \log x_{i}-\beta^{\alpha} \log \beta \sum_{i=1}^{n}\left(\frac{\lambda}{x_{i}}\right)^{\alpha k} \\
& -k \beta^{\alpha} \sum_{i=1}^{n}\left(\frac{\lambda}{x_{i}}\right)^{\alpha k} \log \frac{\lambda}{x_{i}} \\
& \times \frac{n \alpha}{\beta}-\alpha \beta^{\alpha-1} \lambda^{\alpha k} \\
\frac{\partial \log L(x)}{\partial \beta} & \left.=\frac{n \alpha k}{\lambda}-\alpha k \beta^{\alpha} \lambda^{\alpha k-1} x_{i}^{-\alpha k}-n b^{-\alpha k}\right], \\
\frac{\partial \log L(x)}{\partial \lambda} & \times\left[\sum_{i=1}^{n} x_{i}^{-\alpha k}-n b^{-\alpha k}\right], \\
& =\frac{n}{k}+n \alpha \log \lambda-\alpha \beta^{\alpha} \sum_{i=1}^{n}\left(\frac{\lambda}{x_{i}}\right)^{\alpha k} \\
\frac{\partial \log L(x)}{\partial k} & \log \frac{\lambda}{x_{i}}-\alpha \sum_{i=1}^{\alpha}\left(\frac{\lambda}{b}\right)^{\alpha k} \log \frac{\lambda}{b} . \\
& =n x_{i}
\end{aligned}
$$

We can obtain the estimates of unknown parameters by setting the last four equations equal zero, but solving these equations simultaneously to get the unknown parameters $\hat{\alpha}$, $\hat{\beta}, \hat{\lambda}$ and $\hat{k}$ in explicit form is mathematically complicated, so these estimates will be obtained numerically.
The system of equations $\frac{\partial \log L(x)}{\partial \theta_{j}}=0, j=$ $1, \ldots, k$ for parameters vector $\theta$ has a unique root $\hat{\theta} \in(-\infty, \infty)$ if and only if $J(-\infty)>0$ and $J(\infty)<0$, where $J(\theta)=\frac{\partial \log L(x)}{\partial \theta}$. If $\log L(x)$ has multiple local maxima, the highest solution is obtained. For more information, see [4].

\section{Application}

\subsection{Randomly generated data}

A number of thousand random samples were generated for each sample size $\mathrm{n}=50,250$ and 400 by using (7) as $X=\left(b^{-\alpha k}-\right.$ $\left.\beta^{-\alpha} \lambda^{-\alpha k} \ln p\right)^{\frac{-1}{\alpha k}}$ with parameters $\alpha=1.5$, $\beta=0.5, \lambda=0.8$ and $k=1$, and the truncation value $\mathrm{b}=5$, where $\mathrm{u}$ is uniformly distributed. Table 1 shows the estimates, biases and mean squared errors (MSEs) of the parameters for each sample size. It is easy to notice that estimates are close to their actual values with small enough MSE.

Table 1: Estimates, biases and mean squared errors of $\hat{\alpha}, \hat{\beta}, \hat{\lambda}$ and $\hat{k}$

\begin{tabular}{clll}
\hline $\mathrm{n}$ & Estimates & Bias & MSE \\
\hline & $\hat{\alpha}=1.894406$ & 0.3944057 & 0.1555559 \\
50 & $\hat{\beta}=0.5061023$ & 0.006102276 & $3.723777 \times 10^{-5}$ \\
& $\hat{\lambda}=0.9238914$ & 0.1238914 & 0.01534907 \\
& $\hat{k}=0.8823007$ & -0.1176993 & 0.01385312 \\
\hline & $\hat{\alpha}=1.690622$ & 0.1906224 & 0.03633689 \\
250 & $\hat{\beta}=0.4990658$ & -0.0009342476 & $8.728186 \times 10^{-7}$ \\
& $\hat{\lambda}=0.8803825$ & 0.08038254 & 0.006461352 \\
& $\hat{k}=0.9152741$ & -0.08472593 & 0.007178483 \\
\hline & $\hat{\alpha}=1.654117$ & 0.1541169 & 0.02375203 \\
400 & $\hat{\beta}=0.5013534$ & 0.001353353 & $1.831566 \times 10^{-6}$ \\
& $\hat{\lambda}=0.863617$ & 0.06361704 & 0.004047128 \\
& $\hat{k}=0.9250993$ & -0.07490072 & 0.005610118 \\
\hline
\end{tabular}

\subsection{Real-world data}

In this sub section we fitted the RTFWD to some datasets using maximum likelihood estimation and compared the proposed RTFWD with right truncated generalized new extended Weibull 
distribution (RTGNEXWD), right truncated Lindley Weibull distribution (RTLWD) and right truncated half logistic generalized Weibull distribution(RTHLGWD). Their density functions (for $0<x \leq b$ ) are given by

$$
\begin{aligned}
f(x) & =\exp \left(\beta x^{a}-\frac{\sigma}{x^{2}}\right) \\
& \times \frac{\left(2 \theta x+\left(a \beta x^{a-1}+\frac{2 \sigma}{x^{3}}\right) \exp \left(\beta x^{a}-\frac{\sigma}{x^{2}}\right)\right)}{1-\exp \left(-\theta b^{2}-\exp \left(\beta b^{a}-\frac{\sigma}{b^{2}}\right)\right)}, \\
f(x)= & \frac{\beta \theta^{2}\left(\alpha^{\beta} x^{\beta-1}+\alpha^{2 \beta} x^{2 \beta-1}\right) \exp \left(-\theta(\alpha x)^{\beta}\right)}{(\theta+1)\left[1-\exp \left(-\theta(\alpha b)^{\beta}\right)\left(1+\frac{\theta}{\theta+1}(\alpha b)^{\beta}\right)\right]}, \\
f(x) & =\frac{\left(1+\gamma x^{\eta}\right)^{w-1} \exp \left(1-\left(1+\gamma x^{\eta}\right)^{w}\right)}{\left(1-\exp \left(1-\left(1+\gamma b^{\eta}\right)^{w}\right)\right)} \\
& \times \frac{\left(1+\exp \left(1-\left(1+\gamma b^{\eta}\right)^{w}\right)\right) 2 w \eta \gamma x^{\eta-1}}{\left(1+\exp \left(1-\left(1+\gamma x^{\eta}\right)^{w}\right)\right)^{2}}
\end{aligned}
$$

respectively. In order to compare the distributions we calculated the Akaike's information criterion(AIC), the Bayesian information criterion(BIC), Hannan Quinn information criterion(HQIC), Kolmogorov Smirnov (K-S) test, Anderson and Darling $\left(A_{n}^{2}\right)$ test and CramérVon Mises $\left(W^{2}\right)$ test. The model with minimum of these statistics values is chosen as the best model to fit the data. The parameters are estimated by using the maximization of the log-likelihood function and the calculations are performed by using Wolfram Mathematica software.

\subsubsection{Earth quakes dataset}

We will use the dataset earth quakes issued from the datasets R library. This locates the earthquakes off Fiji islands. It gives the locations of 1000 seismic events. The events occurred in a cube near Fiji islands since 1964. The dataset contains 1000 observations of 5 variables: the latitude (lat), longitude (long), Depth in km (depth), magnitude (mag) and the numeric number of stations reporting (stations). Our study will be on magnitude variable and we will take the truncation value of upper whisker limit equals 5.8. The earth quakes dataset is one of the Harvard PRIM-H project datasets. They in turn obtained it from Dr. John Wood house, Dept. of Geophysics, Harvard University.
Table 2 gives the descriptive statistics and table 3 presents the maximum likelihood estimates of the parameters together with the log-likelihood function, AIC, BIC, HQIC, K$\mathrm{S}, A_{n}^{2}$ and $W^{2}$ values for earth quakes dataset after truncation it.

\subsubsection{Life time dataset}

This life time dataset was introduced by Gross and Clark (1975) and it is related to relief times (in minutes) of patients receiving an analgesic. It has been used recently by Shanker, Fesshaye and Selvaraj (2015) to show the flexibility of Exponential distribution and Lindley distribution. We will take the upper whisker limit value as our truncation value which equals 3.075 and the dataset consists of twenty observations

1.1, 1.4, 1.3, 1.7, 1.9, 1.8, 1.6, 2.2, 1.7, 2.7, 4.1, $1.8,1.5,1.2,1.4,3,1.7,2.3,1.6,2$.

Table 4 gives the descriptive statistics and table 5 presents the maximum likelihood estimates of the parameters together with the log-likelihood function, AIC, BIC, HQIC, K$\mathrm{S}, A_{n}^{2}$ and $W^{2}$ values for Life time dataset after truncation it.

From tables (3) and (5), we conclude that the RTFWD behaves best comparable to RTGNEXWD, RTLWD and RTHLGWD distributions for each dataset.
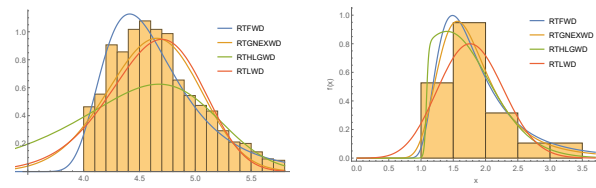

Figure 5: Histogram of datasets and the fitted PDFs

Figure 5 illustrate the histograms and the fitted PDFs of the RTFWD, RTHLGWD, RTGNEXWD and RTLWD, so we conclude that RTFWD behaves best comparable to these distributions. 
Table 2: Descriptive statistics for earth quakes dataset

\begin{tabular}{|c|c|c|c|c|c|c|c|c|}
\hline Min & Max & Mean & Variance & First Quantile & Median & Third Quantile & Skewness & Kurtosis \\
\hline 4 & 5.7 & 4.61037 & 0.148814 & 4.3 & 4.6 & 4.8 & 0.591089 & 2.84355 \\
\hline
\end{tabular}

Table 3: Estimates, Log L, AIC, BIC, HQIC, K-S, $A_{n}^{2}$ and $W^{2}$ for earth quakes dataset

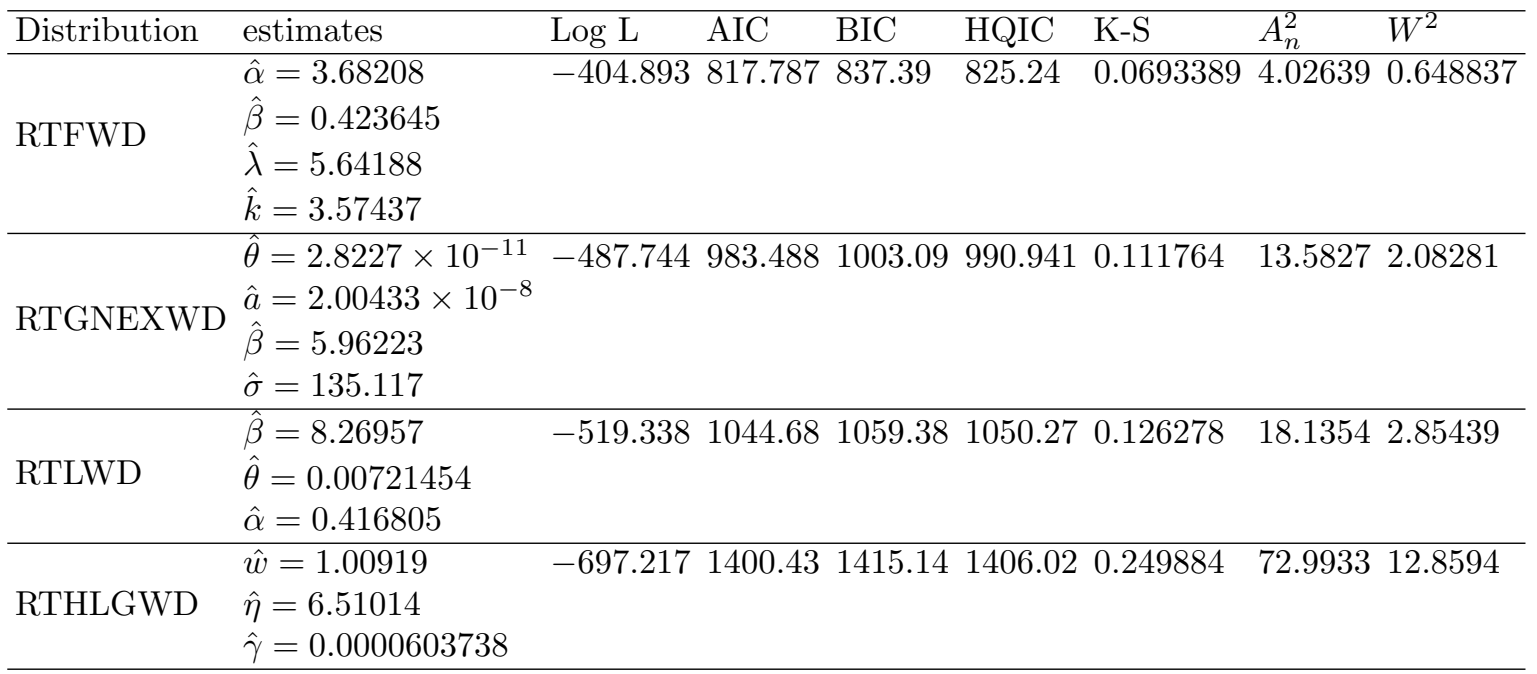

Table 4: Descriptive statistics for second dataset

\begin{tabular}{|c|c|c|c|c|c|c|c|c|}
\hline Min & Max & Mean & Variance & Frist Quantile & Median & Third Quantile & Skewness & Kurtosis \\
\hline 1.1 & 3 & 1.78421 & 0.240292 & 1.425 & 1.7 & 1.975 & 0.970091 & 3.48809 \\
\hline
\end{tabular}

Table 5: Estimates, Log L, AIC, BIC, HQIC, K-S, $A_{n}^{2}$ and $W^{2}$ for life time dataset

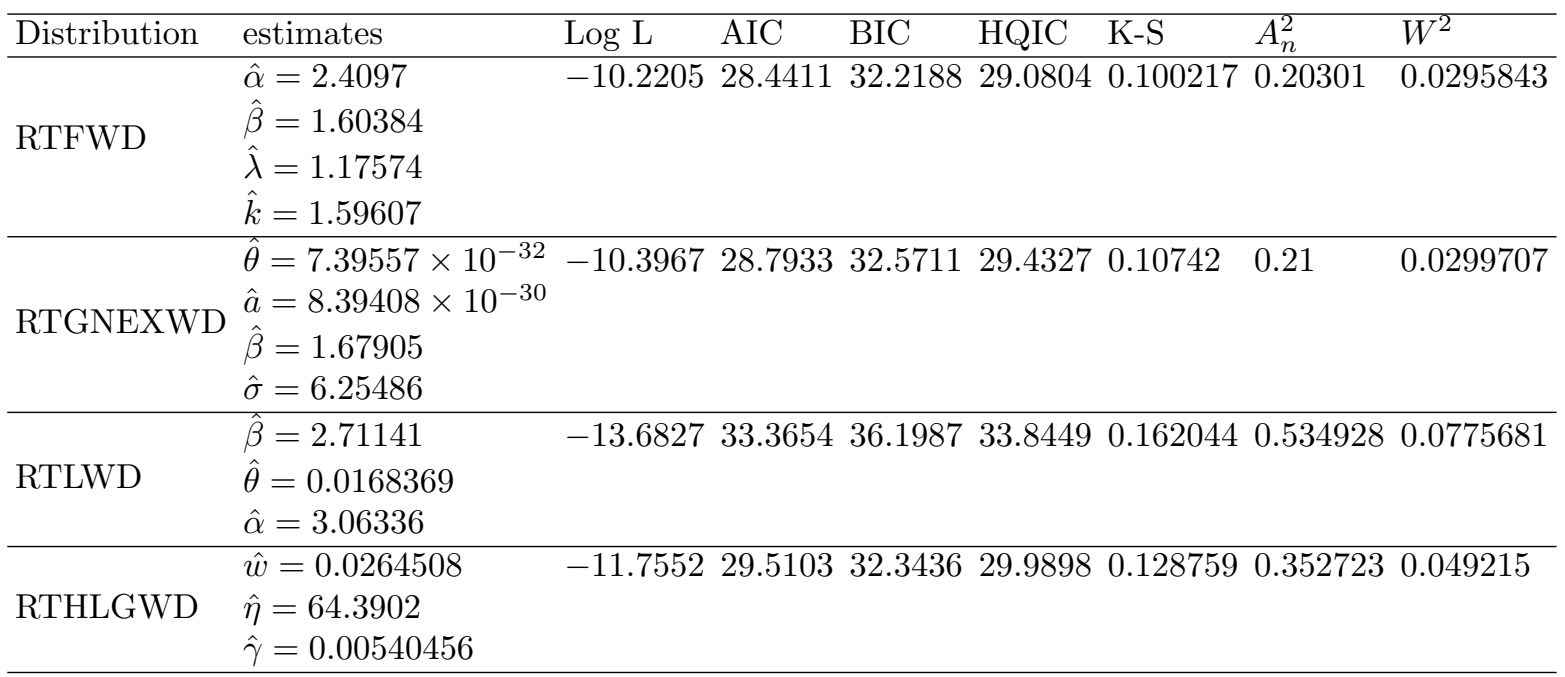
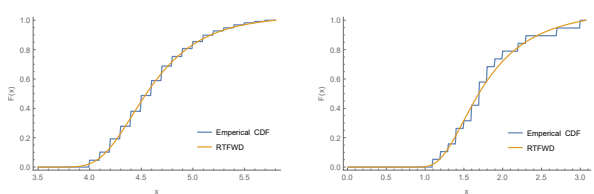

Figure 6: Empirical CDF of datasets and the fitted CDF of RTFWD
Figure 6 shows that our choice for real-world datasets is suitable for RTFWD. 


\section{Conclusion}

This paper introduced a new truncated distribution called RTFWD. Statistical properties of the distribution are studied such as moments, mode, Quantile function,... etc. We also obtained the density function of its order statistics. We calculated the maximum likelihood estimators of the distribution parameters numerically using randomly generated and real-world datasets. The applications to real datasets showed the optimality of our distribution for being better than other compared distributions.

\section{References}

[1] S.H. Abid. Properties of doublytruncated fréchet distribution. American Journal of Applied Mathematics and Statistics, 4(1):9-15, 2016.

[2] Z. Behdani, G. R. M. Borzadaran, and B. S. Gildeh. Some properties of double truncated distributions and their application in view of income inequality. Computational Statistics, pages 1-20, 2019.

[3] M. El-Din, A.A.M. Teamah, A.M.T. Abd El-Bar, and A. Salem. Random sum of mid truncated lindley distribution. $J$. Adv. Res. Stat. Probab, 2(1):27-36, 2010.

[4] M. M. El-Din, Y. Abdel-Aty, and M. H. Abu-Moussa. Statistical inference for the gompertz distribution based on type-ii progressively hybrid censored data. Communications in Statistics-Simulation and Computation, 46(8):6242-6260, 2017.

[5] J. Fellman. Mathematical analysis of distribution and redistribution of income. Science Publishing Group, 2015.

[6] J. Galambos. The asymptotic theory of extreme order statistics. R.E. Krieger Pub. Co., 1987.

[7] J.T. Hattaway. Parameter estimation and hypothesis testing for the truncated normal distribution with applications to introductory statistics grades. Brigham Young University-Provo, 2010.
[8] S. Pundir, S. Arora, and K. Jain. Bonferroni curve and the related statistical inference. Statistics \& probability letters, 75(2):140-150, 2005.

[9] M. S. Tabass, G.R. M. Borzadaran, and M. Amini. Renyi entropy in continuous case is not the limit of discrete case. Mathematical Sciences and Applications e-Notes, 4(1):113-117, 2016.

[10] L. Zaninetti. A right and left truncated gamma distribution with application to the stars. Advanced Studies in Theoretical Physics, 23:1139-1147, 2013. 